\title{
Reckoning the Unresolved Scientific Question on Memory Transfer
}

\author{
Loh Teng-Hern Tan ${ }^{1 \dagger}$, Hooi-Leng Ser ${ }^{1 \dagger}$, Yong Sze Ong ${ }^{2}$ Kooi Yeong Khaw ${ }^{2}$, Siew Hua Gan², Priyia Pus- \\ parajah $^{3}$, Kok-Gan Chan ${ }^{4,5}$, Learn-Han Lee ${ }^{1,6,7^{*}}$, Bey-Hing Goh ${ }^{2,6,8^{*}}$
}

${ }^{1}$ Novel Bacteria and Drug Discovery (NBDD) Research Group, Microbiome and Bioresource Research Strength, Jeffrey Cheah School of Medicine and Health Sciences, Monash University Malaysia, 47500 Bandar Sunway, Selangor Darul Ehsan, Malaysia.

${ }^{2}$ Biofunctional Molecule Exploratory (BMEX) Research Group, School of Pharmacy, Monash University Malaysia, 47500 Bandar Sunway, Selangor Darul Ehsan, Malaysia.

${ }^{3}$ Medical Health and Translational Research Group (MHTR), Jeffrey Cheah School of Medicine and Health Sciences, Monash University Malaysia, 47500 Bandar Sunway, Selangor Darul Ehsan, Malaysia.

${ }^{4}$ Division of Genetics and Molecular Biology, Institute of Biological Sciences, Faculty of Science, University of Malaya, 50603 Kuala Lumpur, Malaysia

${ }^{5}$ International Genome Centre, Jiangsu University, Zhenjiang, China

${ }^{6}$ Health and Well-being Cluster, Global Asia in the 21st Century (GA21) Platform, Monash University Malaysia, Bandar Sunway 47500, Malaysia

${ }^{7}$ Key laboratory of Microbial Metabolism, Joint International Research Laboratory of Metabolic and Developmental Sciences, School of Life Sciences and Biotechnology, Shanghai Jiao Tong University, Shanghai 200240, China

${ }^{8}$ College of Pharmaceutical Sciences, Zhejiang University, 866 Yuhangtang Road, Hangzhou 310058, China

$\dagger$ These authors have contributed equally to this work.

\begin{abstract}
Memory formation occurs within the central nervous system (CNS), specifically in the hippocampal region of brain. The notion that memories are only located within the brain has been challenged by reports of some patients that they have "inherited memories" from their donor after organ transplantation; some even experienced personality changes and picked up hobbies or preferences similar to their donor. Recently, a research team has reignited the embers of this theory by using scientific method to show that memory can be genetically transferred from one sea snail to another. Nevertheless, even as more and more scientific mysteries are being unravelled, memory remains an elusive entity shrouded in the haze of many unresolved hypotheses. To seek clarity on what is currently known, this write-up summarizes and consolidates records associated with the theory of "cellular memory" and experiments evaluating the possibility of memory transference by genetic materials like RNA.
\end{abstract}

Keywords: Memory transfer; engram; RNA; cellular memory; heart transplantation

Received: $6^{\text {th }}$ July 2020

Accepted: $6^{\text {th }}$ August 2020

Published Online: $14^{\text {th }}$ August 2020

*Correspondence: Learn-Han Lee, Jeffrey Cheah School of Medicine and Health Sciences, Monash University Malaysia; lee.learn. han@monash.edu; Bey-Hing Goh, School of Pharmacy, Monash University Malaysia, 47500, Bandar Sunway, Selangor Darul Ehsan, Malaysia; goh.bey.hing@monash.edu

Citation: Tan LT-H, Ser H-L, Yong Sze Ong et al. Reckoning the Unresolved Scientific Question on Memory Transfer. Prog Drug Discov Biomed Sci 2020; 3(1): a0000105. https://doi.org/10.3687/pddbs.a0000105

\section{Introduction}

Do you still vaguely remember the teddy bear that you loved since your childhood? Or even the pair of blue sneakers that you used to cherish when you were going to nursery school? The search for the biological basis of memory is one of the oldest quests in written human history and remains a fascinating enigma ${ }^{[1]}$. "How are the memories formed?", "Where are the memories stored?" and "How long can memories last?" remain as interesting fundamental questions that humans ask and seek to explore. Despite advancement in technology and massive data generated from neuroscientists around the globe, the mechanisms of memory formation and retention remain elusive in neuroscience.

Conventionally, most neuroscience experts believe that memories are generated either by the formation of new 
neurons (neurogenesis) or through the modulation of neurotransmitter release in the synapses between the neurons. Memories are presumably stored in connections between neurons, particularly those residing in the hippocampal region of the brain ${ }^{[2,3]}$. The ability of neurons to communicate is via the release of chemical messengers known as neurotransmitters into the contact point of neurons known as the synapse ${ }^{[4]}$. Upon stimulation, the "sender" or presynaptic neuron relays messages via the release of neurotransmitter, which diffuse through the synaptic gap into the postsynaptic region of "recipient" neuron. This mechanism is similar to the "lock-andkey" hypothesis, whereby the suitable neurotransmitter (key) can unlock and activate the corresponding receptor (lock).

When memory is coupled with emotions, the entire memory formation and retrieval process becomes more sophisticated. Numerous neuroscientists have proven that both humans and animal models tend to relive highly vivid emotional events more frequently than that from mundane experiences ${ }^{[5-7]}$. Emotions can trigger a change in the synaptic strength, activating various brain regions (e.g. amygdala and/or frontal lobe) that may, in turn enhance or diminish memory formation. Subsequently, depending on the number of synapses and/or amount of neurotransmitter and its activated receptors, these factors then determine how long the memory lingers in the brain.

While this model appears to be a satisfactorily neat scientific theory, there is another group of neuroscientists who began to raise questions regarding the dogma of synapses and memory storage, by providing some thoughtprovoking findings ${ }^{[2]}$. Using the sea snail (scientific name: Aplysia californica) as a model, a research group from University of California, Los Angeles (UCLA) has successfully transferred memory from one animal to another by introducing the single-stranded nucleotide chain - ribonucleic acid or RNA extracted from the central nervous system (CNS) of a trained snail into a naïve recipient. Without a doubt, these results daringly challenge the deeply entrenched 'theory' that memories are stored purely through alterations in the strength of synaptic connections between neurons in the brain ${ }^{[8]}$. The experimental design was rather simple — sea snails were trained with mild electrical shocks and their behaviour, namely their siphon withdrawal reflex, was monitored. Those snails which were exposed to electric shocks showed longer duration of siphon withdrawal reflex, a defensive behaviour where the animal would retract its siphon (sensitive part of the body) to protect its gills, compared to untrained snails which were only gently nudged with a probe but did not receive any electrical shock $^{[8]}$. Subsequently, the genetic information in the form of RNA was extracted from the nervous systems of those sensitized or trained (i.e. exposed to electrical shock) snail donors and injected into the body of untrained snail recipients. Much to everyone's surprise, the untrained snail recipients displayed defensive behaviour like their RNA-donor snail; even more fascinating is that this kind of defensive act is only replicated in the snails which received RNA from trained snails and not in those which received RNA from untrained snails. In the same study, the researchers are unable to fully explain the findings that the RNA from trained snails was able to induce long-term sensitization responses in vitro but only in sensory neurons and not motor neurons; when tested on sensorimotor synapses, only a small subset showed longterm sensitization (LTS) potentiation. David Glanzman's group at UCLA therefore propose that memories might be stored within the neuronal nucleus rather than in the synapses, with RNA representing one of the priming components of the engram for long-term sensitization. RNA transfer appears to induce non- synaptic changes that mediate behavioural effect in the sea snail, without altering the DNA sequence. In addition to their most recent findings, Glanzman and his team have previously argued that there is much more to the storage of memory than just formation and maintenance of synapses as they were able to demonstrate that there is persistence of longterm memory in Aplysia which can be restored even after disruption of the new synaptic connections generated during learning. This hypothesis strongly suggests that the memories are not only stored in the synaptic connections, but possibly in the cells too ${ }^{[9]}$. Based on their findings, the research group suggests that the nucleus may be the cellular location of memory storage in sea snails, and RNA appears to have a role in memory formation and consolidation, possibly by inducing epigenetic changes such as DNA methylation and acetylation ${ }^{[8-10]}$ although these findings are not widely accepted by the scientific community.

While Glanzman's theories are very recent, they do inspire a walk down "memory lane", bringing to mind the theories of an unorthodox psychologist in the 1960s, James V. McConnell, who spent years at the University of Michigan attempting to prove that something outside the brain, which he proposed to be 'memory RNA' could be a possible factor for memory transfer. McConnell, who is thought as the founder of the "memory transfer hypothesis"[11], trained flatworms using light flashes and then fed the ground-up bodies of trained flatworms to naive, untrained flatworms. He believed that the recipient cannibalistic worms can gain the knowledge held by the deceased. These cannibal worms proved his hypothesis to be right - the untrained cannibalistic worms appeared to exhibit the behaviour of the trained worms, suggesting that indeed there may be some form of memory transfer between the worms. Moreover, after decapitation, the trained worms were able to regenerate entirely "new" heads and seemed to retain the memory of their previous training. Though the work was replicated by some other laboratories, McConnell's work was largely ridiculed and was often described as a "cautionary tale" because so much time and money was spent by other labs, often ending up in vain with failure, trying to replicate the work $^{[12-14]}$.

In 1990 the scientific community suffered a great loss when McConnell passed away at the age of 65 in Ann Arbor, shortly after completing the sixth edition of his textbook entitled "Understanding Human Behaviour". Although his concept of memory transfer had sparked heated discussion in the scientific world, his achievement was yet to be validated — or at most, partially validated — 
at the time of his passing. This may have been partly due to the fact that technology at that time was not sufficient to extract the requisite evidence. After his demise, the overall acceptability of McConnell's hypothesis slowly faded from scientific discussions until 2013, when a neurosurgeon at Tufts University, Michael Levin, decided to take on McConnell's work to investigate headless flatworms under more controlled settings. He pointed out that McConnell may have indeed been correct and published a research article in 2013 which rejuvenated many of McConnell's ideas about how the creatures' memory work ${ }^{[15]}$.

\section{Could RNA be the engram offering a plausible scientific explanation for memory transfer phenomenon in organ transplantation?}

The concept of memory transfer initiated by McConnell twenty-nine years ago remain to be confirmed: can memories be implanted into a new host via organ transplantation? There have been numerous claims by recipients of organ transplant who acquired emotions, memories and experiences that have been 'transferred' or passed on from their donors. This yet-to-be-proven phenomenon is called "cellular memory". However, as opposed to the theory of connections between neurons, i.e. the synapses as the only site of memory storage, this hypothesis goes beyond the neuron and suggests that non-neuronal human body cells can store memories or information on personalities, tastes, and histories. Due to the mixed opinions between surgeons and scientists, the jury is still out whether or not this is scientifically sound, although there seems to be some willing to give the benefit of doubt to this phenomenon ${ }^{[16,17]}$. However, the vast majority of scientific community remain sceptical as evidenced by some statements made by some medical professionals. "Psychological experience is stored in the brain, it is not something the medical transplant world accepts", John Schroeder, a cardiologist once said ${ }^{[18]}$. Another surgeon, Jeffrey D. Punch wrote that "Organs are not capable of transferring memory to a person's mind in any conventional sense...", once again questioning the cellular memory idea ${ }^{[19]}$. Nevertheless, with the renewed interest and new evidence of the possible role of RNA in serving as a mode of memory transfer as evidenced by Glanzman's finding ${ }^{[8]}$, RNA may be the missing link in providing a scientific basis to this phenomenon, where personal traits may be transferable from one person to another via organ transplantation (Figure 1).

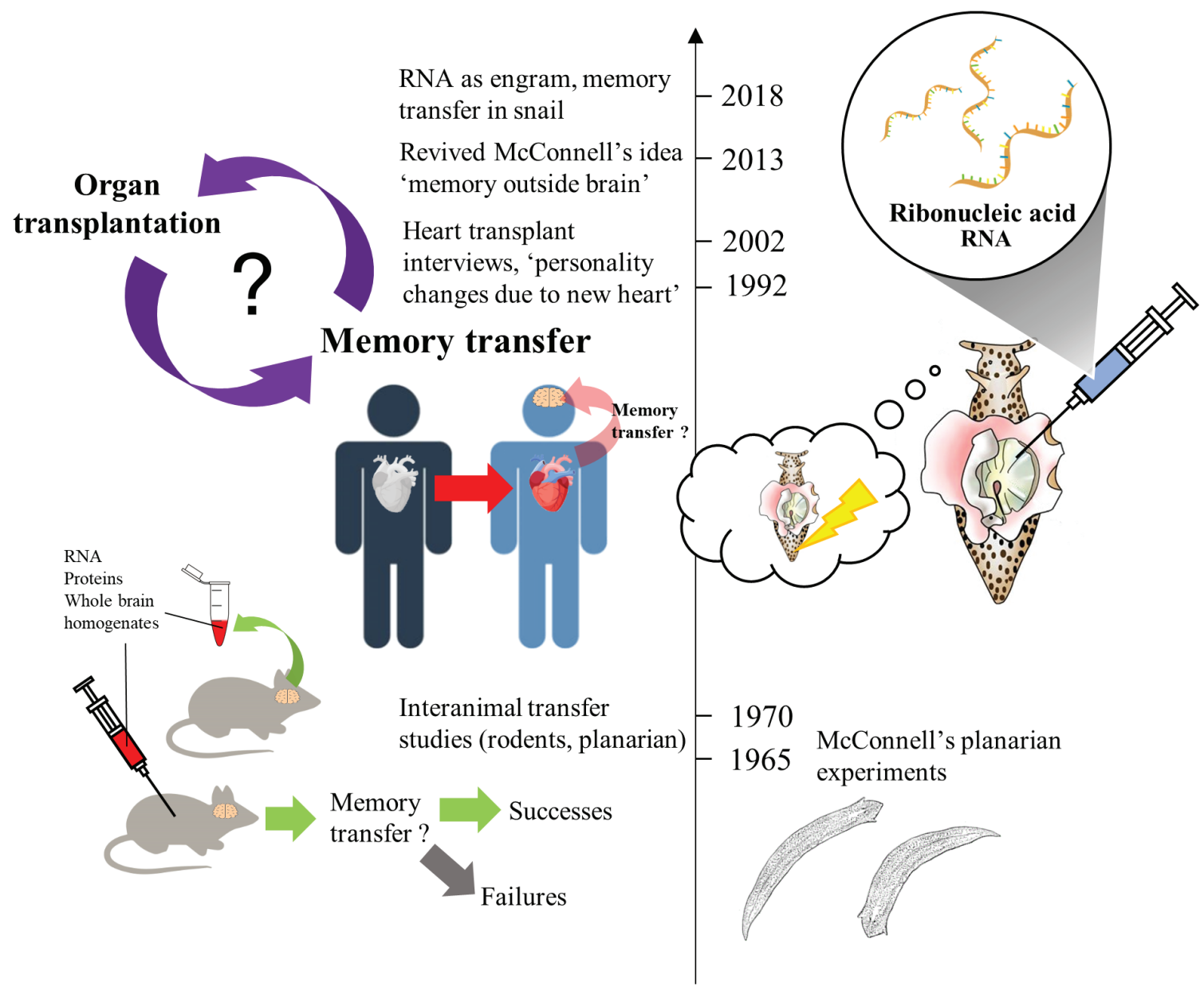

Figure 1. What is the relationship between memory transfer and organ transplantation? Started from the pioneering work by McConnell on flatworms and followed by several supporting works on rodents bolstered the idea of cellular memory and potential role of RNA in transfer of memory. Could be the recent evidence of RNA acted as memory carrier in Aplysia, play a role in explaining the memory transfer phenomena in organ transplant recipients? 
Whilst it is still not considered to be fully scientificallyvalidated, the cellular memory phenomenon does seem to be a plausible explanation for otherwise inexplicable reports of organ transplant receivers claiming that they seem to have "inherited" and acquired the memory, experiences and emotions of their deceased donors, which resulted in noticeable personality changes ${ }^{[19]}$. In fact, most of the studies that support the cellular memory theory rely on anecdotal evidence with a couple of retrospective studies involving a very small sample size/ population of organ transplanted patients ${ }^{[16,20]}$. Pearsall and Schwartz ${ }^{[16]}$ published a study in the Journal of NearDeath Studies which had only ten participants, including a patient only 7 months old at the time of surgery. The study conclusions were drawn from limited evidence, and a closer look revealed an array of possible confounding factors. More often than not, the usual explanations given to patients who experienced personality changes after organ transplantation include the effects of the immunosuppressant drugs, psychosocial stress, and preexisting psychopathology of the recipients ${ }^{[19]}$. Given that it is generally assumed that learning involves primarily the crosstalk between neuronal circuits within the CNS and the immune system, one should not experience personality change parallel to those of donors after receiving peripheral organ transplants ${ }^{[21]}$. Therefore, the assumption for these phenomena is that the behaviours and emotions acquired by the recipient from the original donor might be due to the combinatorial memories stored in the neurons or other cells of the organ donated.

\section{Is there any hidden compartment in the heart for memory?}

Heart transplanted patients are said to be the most likely to experience memory "transfer" or literally, "a change of heart for a change of soul". Apart from Pearsall's team, another study published in Quality of Life Research ${ }^{[22]}$ contained data consolidated from interviews with 47 heart transplant recipients over a two year period in Vienna, Austria. Although the majority of the patients $(79 \%, \mathrm{n}=37)$ did not experience any personality change after the surgery, $15 \%$ of the patients $(n=7)$ noticed a change in personality although not all were attributed to the new organ, while another three patients did notice a drastic change in their personality due to their new hearts. Interestingly, a single 45 years old patient who received a heart from 17 years old boy, claimed to have enjoyed loud music and dreamt of having a car with a good stereo, something he/she did not desire prior to the surgery. While the percentage of personality change as a result of an organ transplant does not seem to be significant, the few reported incidents are fascinating enough to warrant further research to validate the existence of this concept and explain these phenomena.

An additional interesting facet to this mystery is related to the fact that these reported cases of memory transfer seem to be specific to heart transplants. Assuming that the concept of cellular memory is true and accounts for this, what about the myocardial cells or other cells in the heart that facilitates the transfer? Records of kidney transplants and skin grafts procedures can be traced back for decades, well before heart transplants became a reality. However, none of the records indicate any patients describing any phenomena or symptoms that may be related to cellular memory. In addition, successful xenografts and xenotransplants where cells, tissue and organs from another species are transplanted into humans (for example porcine heart valves) also raise questions as to whether cellular memory, if it exists, is species specific, since there have been no reports of humans receiving these implants to have taken on the behavioural characteristic of their donor animal.

At the moment, there are no clear answers to these questions, although some researchers have uncovered some leads which suggest that the heart may have a role in cognitive functions. The work published by Rollin McCraty and his colleagues (HeartMath Research Center in Boulder Creek, California) in 2004 indicated that the heart appears to respond to pre-stimuli even before the brain, and in fact seems to be the path of pre-stimulus information which results in the travel of intuitive behaviour from the heart to the brain ${ }^{[23]}$. Subjects were shown some pictures, some calm and some emotionally arousing pictures, and the heart was shown to demonstrate significant deceleration in response to the arousing images even before actually seeing the image. Therefore, the study suggested that heart may play a direct role in the perception of future events — indirectly implying that the brain may not be the only organ responsible in this regard. McCraty has also published theories on emotional experience which is the result of a dynamic interaction between the heart, CNS and the endocrine systems. At this juncture, things start to become more exciting from the perspective of memory transfer - if the heart truly has a role in intuitive process and generating emotions, it seems feasible that there could be alterations in the heart at a cellular level that have are linked to cognitive function , and when they are transferred from one individual to another, some could be carried along in the process, thus facilitating memory transfer from the donor to the transplant recipient ${ }^{[23-25]}$.

Emotions are described as non-intentional actions that could not be controlled and are usually linked with chemical changes such as the strength of neurotransmitter and hormone signals. It is believed that emotions are somehow associated with health condition, especially in affecting the heart function when the mood changes. A classic example of this effect would be a "broken heart" syndrome or stress-induced cardiomyopathy, whereby some heart compartments are enlarged, associated with temporary disruption in the heart function following a stressful or emotional event. Based on some previous studies, an unusual change of emotions has been observed after heart transplants as if the emotions are transferred simultaneously. Since emotions can affect the function of heart, it may be speculated that the emotions experienced by the heart donor may have elicited epigenetic changes in the genetic codes in the heart cells allowing a memory transfer to occur between two individuals ${ }^{[26,27]}$.

To date, several theories have been put forward to explain the possible links between the heart and the brain involved in the memory process. Dr. J. Andrew Armour first introduced the concept of a functional 'heart 
brain'. Apart from its role as a physical pump from the anatomical point of view, the heart also contains an intrinsic nervous system that exhibits both short and long-term memory functions. Based on his analysis, Dr Armour described that the intrinsic nervous system of the heart consists of approximately 40,000 neurons called sensory neurites which are responsible for relaying afferent information to the brain ${ }^{[28]}$. It is possible that the transfer of information represents a newly discovered center of intelligence which may play a pivotal part in memory transfer. In actual fact, it was observed that the heart communicates with the brain in ways that significantly affect how a person perceives and reacts to the world. Now seen as a highly complex, self-organizing information processing center, the heart has its own functional 'brain' that influences and communicates with the cranial brain via the nervous system (through transmission of nerve impulses), hormonal system (hormones and neurotransmitter) ${ }^{[29]}$ and other pathways including pressure waves and electromagnetic field ${ }^{[30]}$. Recently, a neurocardiology research has also revealed the complex neural interactions between the heart and the brain, of which the afferent information processed by the intrinsic cardiac nervous system can influence perception and cognitive functions of the brain ${ }^{[31]}$.

\section{Discussions}

Sir Isaac Newton once said "If I have seen further, it is by standing on the shoulders of giants" [32]. This quote is truly inspiring for those in the scientific community to explore and find answers for unexplained phenomena. Over the past few decades, neuroscience research has grown tremendously from experiments on simple organisms like flatworms and sea snails to meticulous cell culture studies that require isolation of a single type of neuronal population from animals or even humans - with all these efforts working towards the elusive goal of elucidating the biological mechanisms underlying the learning and memory processes. Even with all the effort so far, neuroscientists have only begun to get a glimpse on the process of short and/or long-term memory formation with the core theory that it involves changes in synaptic strength and modification of individual neurons during learning ${ }^{[33,34]}$. The recent dramatic improvements in technologies like functional imaging and electrophysiological monitoring along with a booming computational power allow researchers to get closer to the aim of delineating the structure of neural circuits in more complex forms of explicit memory ${ }^{[35,36]}$. However, these are only largely able to visualize or indirectly demonstrate the circuits of conventional neuroscience. It is uncertain if even these tools are sufficient in confirming the concepts raised by McConnell's and Glanzman's teams - and their claims, if proven to be correct, could essentially revolutionize the neuroscience field, by completely transforming what we have learnt all these while about cognitive processes.

Apart from synapses, researchers are finding other possible factors or mechanisms that may be involved in memory storage and transfer. All these years, although animals like the sea snails and flatworms have been confirmed to be a powerful model organism for neuroscience, but sadly, the brains of these organisms which are far simpler work differently than that of humans. Thus, it is vital to consider replicating these experiments in animals with more complex brains or those with a higher cognitive function. By the same token, there was some earlier work reported in the 1960s displaying memory transfer via RNA in mammals including rats and hamsters ${ }^{[37-39]}$. These findings were consistent with what McConnell had observed in flatworms; with one of the studies even demonstrating memory transfer when they conducted a cross species test (i.e. injecting rat RNA to hamster and vice versa).

If memories are contained in the nuclei as RNA of specific brain cells or even the cardiac cells, it would be at least theoretically possible to "pack" these RNA memories into "memory capsules" and inject them on demand which could make a massive impact on some treatment modalities like for dementia and Alzheimer's disease. What we know currently about memory formation is that it requires activation of certain genes which lead to specific protein synthesis, in which RNA plays a crucial role as signalling molecule or "messenger" to kick start and regulate these processes. Based on what Glanzman's team has proposed, if RNA molecules present in the donor organ possess some "secret codes" that can be translated into memory, it could explain the observations in those organ transplant recipients who experienced major changes in their attitude or even having blurry memories inherited from their organ donor. Therefore, if these proteins can be isolated and identified, it is safe to say that the potential applications would be beyond the wildest dreams of neurologists and neuroscientists alike, since the concept of "memory capsules" can pose as a possible treatment for memory loss - a debilitating condition that severely interferes with the daily lives of many, especially in the elderly. With the concept of storing and transferring memory, patients with severe depression, Alzheimer's and amnesia could presumably have some of part of their memories restored. However, this concept may give rise to a controversy since theoretically it would also allow someone to easily implant false and distorted memories.

From the technical perspective, if RNA can encode for memory, what would be the best way to administer these "memory capsules" into the host? At the moment, by using suitable packing materials, recent advancements in drug delivery system can precisely transport drugs across one of the most problematic barriers separating the brain and the rest of the body or the blood-brain barrier. For example, the promising results revealed by Matthew J. A. Wood and colleagues, successfully introduced exosomes containing RNA sequence intravenously that can make their way across the blood brain barrier to switch off a gene implicated in Alzheimer's disease ${ }^{[40]}$. Perhaps, this can assist in designing the appropriate bullet for memory transfer - by packing RNA into exosomes and evaluating their ability to induce behavioural changes in untrained animals. Also, provided that the in-built complexity in memory formation (which can be segregated into acquisition, consolidation and information retrieval), as well as the different types and composition of RNA, it is worth mentioning that these are the major factors 
limiting the outcome of significant scientific research in an explicit manner. All in all, it is still necessary for these studies to be conducted in non-human primate models for validation before introducing the methods into clinical settings. Nevertheless, just like any other scientific projects, there will be other considerations whether to further substantiate this idea in future work, particularly ethical constraints and moral concerns when it comes to the use of non-human primates for brain and behaviour studies ${ }^{[4-43]}$. From the little evidence shown in the effort to delineate how memory could be transferred, this reflects that there is still a long way to go before we get to figure outthe mechanisms behind these phenomena. Keeping in mind that it may still be wise to proceed with caution, researchers could still make use of these findings as a foundation and take this research to a higher level. Just like what Glanzman claims ${ }^{[8]}$, knowing the neural substrate of memory could be a crucial cornerstone for future therapeutic applications of memory manipulation, thereby it could offer tremendous potential for future treatment of memory-related disorders, including restoring lost, precious memories in Alzheimer's disease patients or even reducing emotional pain by deleting undesirable memories in post-traumatic stress disorder (PTSD) patients.

\section{Conclusions}

The renewed interest and new evidence of the possible role of RNA in serving as a mode of memory transfer as evidenced by Glanzman's finding, RNA may be the missing link in providing a scientific basis to this phenomenon, where personal traits may be transferable from one person to another via organ transplantation. Whether the concept of memory transfer remains as a fictional tale or actual facts, the quest remains and new technology is now available for scientists to refresh our current understanding and attain the new knowledge on memory formation processes, in parts other than CNS. Even though many individuals may feel that the current progress towards explanation of learning and memory has reached stagnation point, it is important to always bear in mind that with the rapid evolution in molecular techniques and visualization tools, neuroscientists may be able to extend their exploration towards alternative hypothesis, in the search for answers to these unsolved science mysteries. Perhaps, now is the right time to seriously reconsider theories that vanished after nearly half a century ago. Hence, in this regard, the inconclusive claims from the role of RNA in memory transfer experiments merit further investigation in context of newer advancements in neuroscience.

\section{Author Contributions}

The writing was performed by LT-HT, H-LS, O-YS, K-YK, S-HG, PP, K-GC, L-HL and B-HG. While B-HG provided vital guidance and insight to the work. The project was conceptualized by B-HG.

\section{Conflict of interest}

The authors declare no conflict of interest.

\section{Acknowledgement}

This research was funded by Monash Global Asia in the 21st Century (GA21) research grant (GA- HW-19-L01 \& GA-HW-19-S02).

\section{Reference}

1. Queenan BN, Ryan TJ, Gazzaniga MS, et al., On the research of time past: the hunt for the substrate of memory. Ann N Y Acad Sci 2017; 1396(1): 108-125.

2. Sossin WS, Memory synapses are defined by distinct molecular complexes: A proposal. Front Synaptic Neurosci 2018; 10: 5.

3. Mayford M, Siegelbaum SA, and Kandel ER, Synapses and memory storage. Cold Spring Harb Perspect Biol 2012; 4(6): a005751.

4. Fields RD, Making memories stick. Sci Am 2005; 292(2): 75-81.

5. Buchanan TW, Retrieval of emotional memories. Psychol Bull 2007; 133(5): 761.

6. Clewett D and Murty VP, Echoes of emotions past: How neuromodulators determine what we recollect. eNeuro 2019; 6(2).

7. Chen BK, Murawski NJ, Cincotta C, et al., Artificially enhancing and suppressing hippocampus-mediated memories. Curr Biol 2019.

8. Bédécarrats A, Chen S, Pearce K, et al., RNA from trained Aplysia can induce an epigenetic engram for long-term sensitization in untrained Aplysia. eNeuro 2018; 5(3): ENEURO. 0038-18.2018.

9. Chen S, Cai D, Pearce K, et al., Reinstatement of long-term memory following erasure of its behavioral and synaptic expression in Aplysia. Elife 2014; 3.

10. Pearce K, Cai D, Roberts AC, et al., Role of protein synthesis and DNA methylation in the consolidation and maintenance of long-term memory in Aplysia. Elife 2017; 6 .

11. Larry S. The memory-transfer episode [Internet]. 2010 [Cited 9 July 2020]. Available from: https://www.apa.org/monitor/2010/06/memorytransfer.

12. Hartry AL, Keith-Lee P, and Morton WD, Planaria: Memory transfer through cannibalism reexamined. Science 1964.

13. Walker D, Memory transfer in planarians: an artifact of the experimental variables. Psychon Sci 1966; 5(9): 357-358.

14. Weigmann K, The consequence of errors: From memory molecules to the criminal chromosome, erroneous conclusions continue to blight scientific research. EMBO Rep 2005; 6(4): 306-309.

15. Shomrat $\mathrm{T}$ and Levin $\mathrm{M}, \mathrm{An}$ automated training paradigm reveals longterm memory in planarians and its persistence through head regeneration. J Exp Biol 2013; 216(20): 3799- 3810.

16. Pearsall P, Schwartz GE, and Russek LG, Changes in heart transplant recipients that parallel the personalities of their donors. J Near-Death Stud 2002; 20(3): 191-206.

17. Pearsall PP. The heart's code: Tapping the wisdom and power of our heart energy. Harmony; 1999.

18. Carol R and Carol R, The skeptic's dictionary: A collection of strange beliefs, amusing deceptions, and dangerous delusions. 2003, New York: Wiley.

19. Dossey L, Transplants, cellular memory, and reincarnation. Explore (NY) 2008; 4(5): 285-293.

20. Pearsall P, Schwartz GE, and Russek LG, Organ transplants and cellular memories. Nexus Magazine 2005; 12(3).

21. Marin I and Kipnis J, Learning and memory... and the immune system. Learn Mem2013; 20(10): 601-606.

22. Bunzel B, Schmidl-Mohl B, Grundböck A, et al., Does changing the heart mean changing personality? A retrospective inquiry on 47 heart transplant patients. Qual Life Res 1992; 1(4): 251-256.

23. McCraty R, Atkinson M, and Bradley RT, Electrophysiological evidence of intuition: Part

24. The surprising role of the heart. J Altern Complement Med 2004; 10(1): 133-143.

25. Brosschot JF and Thayer JF, Heart rate response is longer after negative emotions than after positive emotions. Int J Psychophysiol 2003; 50(3): 181-187.

26. Meerson F, Arkhipenko I, Rozhitskaia I, et al., Damage to the Ca2+transport system of cardiac sarcoplasmic reticulum during emotion-pain stress. Biull Eksp Biol Med 1981; 91(4): 405-406.

27. Gaudi S, Guffanti G, Fallon J, et al., Epigenetic mechanisms and as- 
sociated brain circuits in the regulation of positive emotions: A role for transposable elements. J Comp Neurol 2016; 524(15): 2944-2954.

28. Pitkanen M, Could RNA \& protein methylation of RNA be involved with the expression of molecular emotions? J Conscious Explor Res 2019; 9(9).

29. Armour J, Anatomy and function of the intrathoracic neurons regulating the mammalian heart. Reflex control of the circulation, ed. I.H. Zucker and J.P. Gilmore. 1991, Boca Raton, Florida: CRC Press, USA. $1-37$.

30. Cantin M and Genest J, The heart as an endocrine gland. Sci Am 1986; 254(2): 76-81

31. McCraty R, Atkinson M, Tomasino D, et al., The coherent heart heartbrain interactions, psychophysiological coherence, and the emergence of system-wide order. Integral Review: A Transdisciplinary \& Transcultural Journal for New Thought, Research, \& Praxis 2009; 5(2).

32. Shaffer F, McCraty R, and Zerr CL, A healthy heart is not a metronome: an integrative review of the heart's anatomy and heart rate variability. Front Psychol 2014; 5: 1040.

33. Poston GJ, Standing on the shoulders of giants. Eur J Surg Oncol 2008; 34(3): 253-255.

34. Bliss TV and Collingridge GL, A synaptic model of memory: long-term potentiation in the hippocampus. Nature 1993; 361(6407): 31.

35. Takeuchi T, Duszkiewicz AJ, and Morris RG, The synaptic plasticity and memory hypothesis: encoding, storage and persistence. Philos
Trans R Soc B Biol Sci 2014; 369(1633): 20130288.

36. Kandel ER, Dudai Y, and Mayford MR, The molecular and systems biology of memory. Cell 2014; 157(1): 163-186.

37. Alberini CM and Kandel ER, The regulation of transcription in memory consolidation. Cold Spring Harb Perspect Biol 2015; 7(1): a021741.

38. Babich FR, Jacobson AL, Bubash S, et al., Transfer of a response to naive rats by injection of ribonucleic acid extracted from trained rats. Science 1965; 149(3684): 656-657.

39. Babich FR, Jacobson AL, and Bubash S, Cross-species transfer of learning: effect of ribonucleic acid from hamsters on rat behavior. Proc Natl Acad Sci 1965; 54(5): 1299- 1302.

40. Fjerdingstad E, Nissen T, and Røigaard-Petersen H, Effect of ribonucleic acid (RNA) extracted from the brain of trained animals on learning in rats. Scand J Psychol 1965; 6(3): 1-6.

41. Alvarez-Erviti L, Seow Y, Yin H, et al., Delivery of siRNA to the mouse brain by systemic injection of targeted exosomes. Nat Biotechnol 2011; 29(4): 341

42. Greene M, Schill K, Takahashi S, et al., Moral issues of human-non-human primate neural grafting. Science 2005; 309(5733): 385-386.

43. DeGrazia D, Nonhuman primates, human need, and ethical constraints. Hastings Cent Rep 2016; 46(4): 27-28.

44. Neuhaus CP, Ethical issues when modelling brain disorders innon-human primates. J Med Ethics 2018; 44(5): 323-327. 